\title{
Forecasting the Need for Dialysis Services in Ontario, Canada to 2011
}

\section{Prévision des besoins en services de dialyse jusqu'en 2011 en Ontario, Canada}

\author{
इำ \\ ROBERT R. QUINN, MD \\ Institute for Clinical Evaluative Sciences \\ Department of Medicine, University of Toronto \\ Clinical Associate, Division of Nephrology \\ Sunnybrook Health Sciences Centre \\ Toronto, ON
}

ANDREAS LAUPACIS, MD, MSC

Keenan Research Centre at the Li Ka Shing Knowledge Institute of St. Michael's Hospital Professor, Faculty of Medicine, University of Toronto

Toronto, ON

JANET E. HUX, MD, MSC

Associate Professor, Department of Health Policy, Management and Evaluation, University of Toronto

President and CEO(I), Senior Scientist, Institute for Clinical Evaluative Sciences Sunnybrook Health Sciences Centre Toronto, ON

\section{RAHIM MOINEDDIN, PHD}

Institute for Clinical Evaluative Sciences

Assistant Professor, Department of Public Health Sciences and Department of Family and Community Medicine, University of Toronto Toronto, ON 
Robert R. Quinn et al.

MICHAEL PATERSON, MSC

Lead, Drugs, Diabetes and Kidney Research Program

Institute for Clinical Evaluative Sciences

Assistant Professor, Department of Family Medicine, McMaster University

Hamilton, ON

MATTHEW J. OLIVER, MD, MHS

Assistant Professor, Department of Medicine and

Department of Health Policy, Management and Evaluation, University of Toronto

Medical Director, Chronic Dialysis

Sunnybrook Health Sciences Centre

Toronto, ON

\begin{abstract}
Careful projections of the demand for dialysis services are important to assist healthcare planners in forecasting the need for equipment, facilities and personnel. We used time series techniques to model the historical incidence and prevalence counts and to forecast the predicted number of patients requiring dialysis in the province of Ontario to 2011. We showed that the incidence and prevalence of dialysis patients continues to grow rapidly. More importantly, traditional definitions of "chronic dialysis" capture only $52 \%$ of all incident patients and ignore the acute dialysis population. Projections about the need for dialysis services based on these definitions may result in underestimation of the resources required to care for the end-stage renal disease (ESRD) population.
\end{abstract}

\title{
Résumé
}

Une projection consciencieuse de la demande pour les services de dialyse est cruciale pour aider les planificateurs de la santé dans leurs prévisions en matière d'équipement, d'installation et de personnel. Nous avons employé la technique des séries chronologiques pour créer un modèle de l'incidence et de la prévalence et pour prévoir le nombre de patients ontariens qui auront besoin de services de dialyse jusqu'en 2011. Nous démontrons que l'incidence et la prévalence en matière de dialyse continuent de croître rapidement. De plus, la définition traditionnelle de la « dialyse pour les cas chroniques » ne correspond quà $52 \%$ de toutes les incidences et ne tient pas compte des cas de dialyses d'urgence. Les projections qui se fondent sur cette définition pourraient mener à une sous-estimation des ressources nécessaires pour traiter les cas d'insuffisances rénales terminales. 
$\mathrm{P}$ ATIENTS With end-STAge Renal disease (ESRD) have A Reduced Quality of life (Evans et al. 1985; Laupacis et al. 1996) and a high mortality rate (CIHI 2006), and consume a disproportionate amount of healthcare resources (USRDS 2006). The number of ESRD patients worldwide is growing rapidly, and they are currently responsible for $5 \%$ to $7 \%$ of healthcare expenditures in developed countries (De Vecchi et al. 1999; USRDS 2006). Careful projections of the demand for dialysis services are important to assist healthcare planners in forecasting the need for equipment, facilities and personnel.

Previous studies that have attempted to predict the growth in the ESRD population have restricted their analyses to chronic dialysis patients (Farrow et al. 1972; Murakami and Ohashi 2001; Schaubel et al. 2001; Xue et al. 2001; You et al. 2002), often defined as patients who are alive on therapy 90 days after the initial treatment. However, this approach may not be adequate to inform resource allocation and planning, as it underestimates the number of patients that require treatment at a given time and does not account for the acute dialysis population. The objective of this study was to identify all patients who received at least one dialysis treatment in Ontario, Canada and to describe the disposition of this cohort 90 days following the initiation of therapy. We then used time series analysis to model historical incidence and prevalence data and to make projections about the need for dialysis services in the province to 2011. Finally, we determined the proportion of dialysis activity that was attributable to hospitalized patients with acute renal failure in an attempt to quantify the potential impact of this group on resource utilization.

\section{Subjects and Methods \\ Patient population}

All incident and prevalent dialysis patients in Ontario were identified between July 1 , 1998 and September 30, 2005 using administrative health data housed at the Institute for Clinical Evaluative Sciences (ICES). We identified anyone who had received at least one dialysis treatment during the study period, including patients who had a remote history of temporary treatment with dialysis and those who had received a previous kidney transplant.

\section{Data sources}

Dialysis patients were identified using physician billing claims in the Ontario Health Insurance Plan (OHIP) database. An OHIP claim was submitted for each treatment (up to a maximum of four treatments) in acute dialysis patients and on a weekly basis for chronic dialysis patients. Billing claims included information about the type of 
therapy - peritoneal dialysis $(\mathrm{PD})$ versus haemodialysis $(\mathrm{HD})$ - being received after July 1, 1998 (see Appendix A). Dialysis providers in one region of the province were reimbursed under an alternative funding arrangement and, as a result, billing claims in that region were unreliable. The number of prevalent dialysis patients at each of the dialysis centres in that region was collected by review of patient charts and dialysis unit records. Prevalent counts were used to determine the current rate of dialysis per million population (PMP). We assumed that the rate of dialysis in that region had a constant relationship with the provincial rate over the duration of the study period and used it to generate expected counts.

The Registered Persons Database (RPDB) was used to obtain demographic and vital status information and to determine the total population of patients who were eligible for OHIP coverage. The prevalence of diabetes in the province was determined using the previously validated Ontario Diabetes Database (ODD) (ICES 2003).

Information concerning hospitalizations and kidney transplantation were taken from the Canadian Institute for Health Information Discharge Abstracts Database (CIHI-DAD). Hospitalization data were used to determine the proportion of patients starting dialysis in an acute care facility. The start of outpatient dialysis was defined by the date of discharge from the patients' initial hospitalization. Dialysis treatment periods ended upon patients' receipt of a kidney transplant.

\section{Statistical analysis}

All patients who had received at least one dialysis treatment during the study period were followed for 90 days after the initiation of therapy. We described the distribution of initial dialysis treatment modalities, the proportion of patients who started dialysis in hospital and the proportion that required outpatient dialysis at some point during follow-up. In addition, we reported the disposition of all patients 90 days after their first treatment (alive and no longer requiring dialysis, alive on dialysis or dead).

The number of patients requiring dialysis therapy was determined at regularly spaced, three-month intervals for the entire study period. Incident patients were defined as all individuals who had their first dialysis billing claim during the quarter. All patients who had a claim for dialysis in the preceding 28 days and who were alive on the first day of the period of interest were included in prevalent counts ("All Prevalent Patients"). Prevalent patients were then labelled "Prevalent Outpatients" if they had received dialysis in an outpatient facility at any point during their treatment history and were further subdivided according to treatment modality ("Prevalent Outpatient Haemodialysis [HD]" and "Prevalent Outpatient Peritoneal Dialysis [PD]").

Time series techniques were used to model the historical incidence and prevalence counts. An exponential smoothing model, auto-regressive integrated moving average (ARIMA) model, stepwise auto-regressive model and an exponential smoothing 
model using Winter's method were fitted to each variable of interest. Plots of observed and predicted values were examined for evidence of fit and objective measures, including $R^{2}$ (the proportion of the variance explained by the model) and the mean percentage error, were calculated. The models were then used to forecast the predicted number of incident and prevalent patients requiring dialysis in the province to 2011 . The absolute numbers of patients were reported, as well as the rates of dialysis per million population using population estimates from the RPDB. All analyses were performed using SAS, Version 9.1 (Cary, NC).

\section{Results}

\section{Population demographics and diabetes prevalence}

The total population of OHIP-eligible individuals in the province grew from 10.6 million in 1998 to 11.4 million by the end of 2005. The mean age of the population increased from 43.4 years to 45.6 years over the study period. An estimated $7.2 \%$ of the adult general population had a diagnosis of diabetes mellitus in 2005 compared to just $4.5 \%$ in 1998.

\section{New dialysis patients}

A total of 31,679 new dialysis patients were identified between July 1, 1998 and September 30, 2005. Seventy-three per cent received HD as their initial form of dialysis treatment, $15 \%$ started on some form of continuous renal replacement therapy (CRRT) and $12 \%$ started on PD. Of all new patients, $62 \%$ started dialysis in hospital, and $27 \%$ of this cohort died prior to discharge. The disposition of incident patients, 90 days following the initiation of dialysis, is presented in Table 1 . Sixty-three per cent of new dialysis patients went on to require treatment in an outpatient dialysis unit at some point during follow-up.

TABLE 1. Outcomes of incident dialysis patients, overall and by first treatment modality

\begin{tabular}{|l|l|l|l|l|}
\hline \multicolumn{2}{|c|}{} & \multicolumn{3}{c|}{ Status at 3 Months } \\
\hline Initial modality & $\begin{array}{l}\text { Ever received } \\
\text { outpatient dialysis }\end{array}$ & Dead & Alive on dialysis & $\begin{array}{l}\text { Recovered kidney } \\
\text { function* }\end{array}$ \\
\hline CRRT $(N=4,793)$ & $15 \%$ & $53 \%$ & $8 \%$ & $39 \%$ \\
\hline HD (N=22,98I) & $68 \%$ & $19 \%$ & $56 \%$ & $25 \%$ \\
\hline PD (N=3,905) & $95 \%$ & $6 \%$ & $83 \%$ & $11 \%$ \\
\hline $\begin{array}{l}\text { All incident patients } \\
(N=31,679)\end{array}$ & $63 \%$ & $23 \%$ & $52 \%$ & $25 \%$ \\
\hline
\end{tabular}

CRRT = Continuous Renal Replacement Therapy; HD = Haemodialysis; PD = Peritoneal Dialysis 


\section{Incidence and prevalence over time}

Between 1998 and 2005, the average annual growth in the incident dialysis population was $4.9 \%$. The incidence rate increased from 351 patients PMP to 458 patients PMP over the same period (Figure 1). The growth in the prevalent dialysis population outpaced incidence, growing an average of 7.2\% annually to reach 8,411 patients by 2005 . The prevalence of outpatient dialysis climbed from 497 patients PMP in 1998 to 737 patients PMP by 2005 (Figure 1) and the proportion of prevalent outpatients being treated with PD at that time was $18 \%$.

FIGURE 1. Historical incidence and prevalence rates, per million population (PMP)

\begin{tabular}{|l|l|l|l|l|l|l|}
\hline & \\
\hline
\end{tabular}

$\mathrm{HD}=$ Haemodialysis; $\mathrm{PD}=$ Peritoneal Dialysis

*To determine whether an individual meets criteria for this category requires additional follow-up time; values are not reported for 2005. Notes:

Population estimates were taken from the Registered Persons Database (RPDB) and reflect the number of individuals eligible for OHIP coverage in the province of Ontario in a given year. The prevalence of ESRD patients requiring dialysis therapy continues to rise, driven predominantly by the growth in the outpatient haemodialysis population. "Incident Outpatient Dialysis" refers to the rate of new starts that go on to require treatment as an outpatient. "Incident 90-Day Dialysis" refers to those incident patients who are alive on dialysis 90 days following the start of treatment.

\section{Forecasts of incidence and prevalence}

Stepwise auto-regressive models demonstrated the best fit to our data $\left(R^{2}=0.883\right.$ to 0.999 ; mean percentage error -0.448 to 0.066 ) and allowed the most precise forecasts of the projected need for dialysis services. Figure 2 shows the actual historical data, the predicted values according to our models and the forecasted incidence and prevalence. We estimate that over 4,000 new patients will require care in an outpatient dialysis unit in the year 2010. The total number of dialysis patients being cared for in 
the province of Ontario is expected to grow by a mean annual rate of $5.5 \%$ and reach $11,104$ ( $95 \%$ confidence interval [CI]: 10,931 to 11,277$)$ by 2011 . The prevalent outpatient population will be an estimated 10,796 patients (95\% CI: 10,655 to 10,938), and $85 \%$ will be treated with haemodialysis if current trends continue.

\section{Impact of including acute inpatient dialysis on growth projections}

Prevalent outpatients, defined as those individuals who received at least one week of outpatient dialysis therapy at some point during follow-up, accounted for an average of $97 \%$ of the total prevalent population. Thus, at any given time, $3 \%$ of all patients being treated were patients who started acute dialysis in hospital and had not yet made it to discharge.

FIGURE 2. Historical incidence and prevalence data with forecasts to $201 \mathrm{I}$

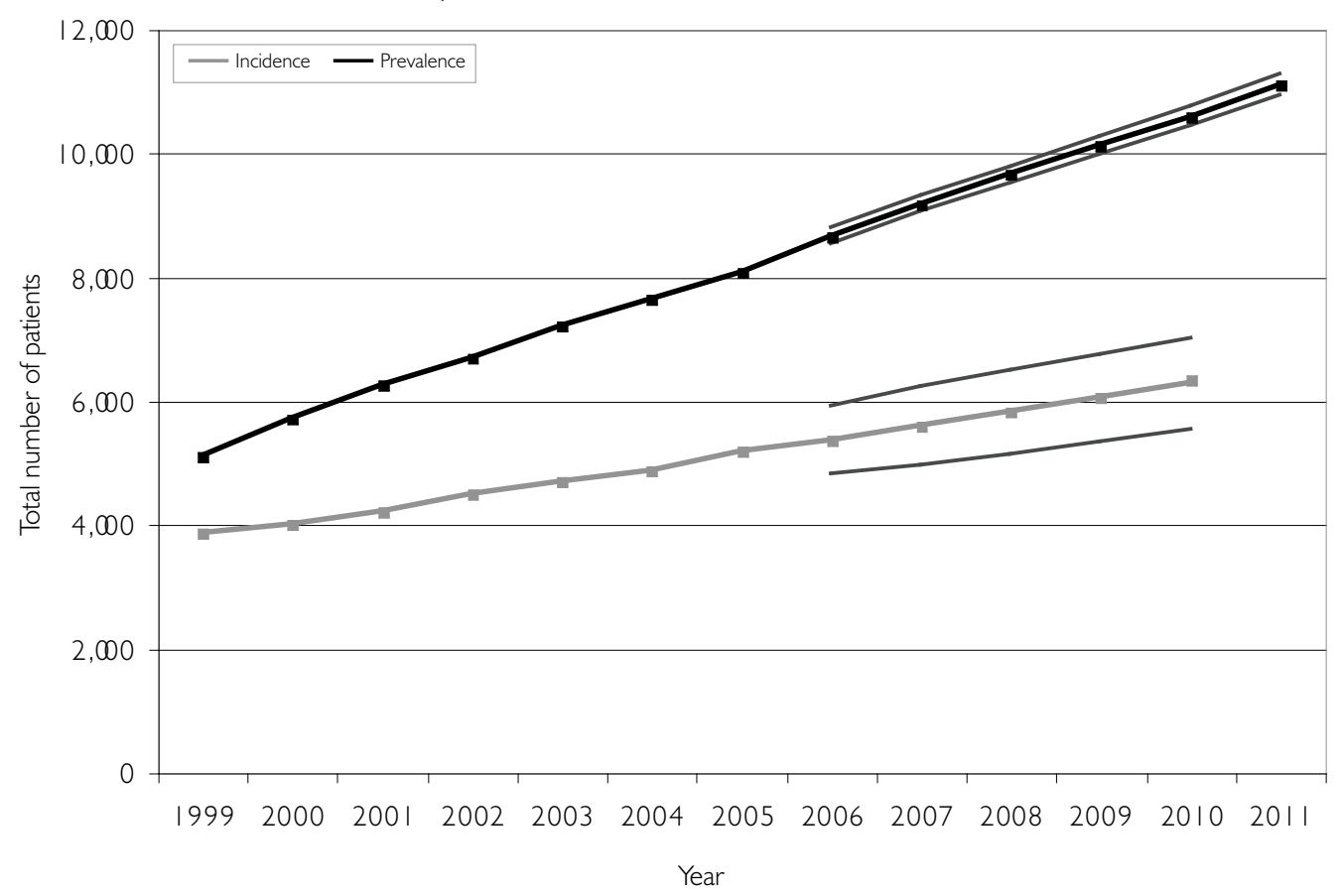

Notes:

The annual number of incident and point prevalent dialysis patients is plotted between 1999 and 2005. Projections of the number of new patients requiring dialysis treatment in the province for 2006 to 2010 are shown with accompanying $95 \%$ confidence intervals. During the year 2010 , an estimated 6,28I new patients (95\% confidence limits: 5,542 to 7,020) will require dialysis for the first time. Forecasts of the point prevalent dialysis population in the province to $201 \mathrm{I}$ are shown with accompanying $95 \%$ confidence intervals. It is estimated that the number of patients requiring dialysis therapy will grow by an average of $5.5 \%$ per year to reach II, I04 (95\% confidence limits: I0,93। to II,277) by 2011 if current trends continue. 


\section{Discussion}

We used physician billing claims to identify 31,679 patients who received dialysis in the province of Ontario between 1998 and 2005. More than $60 \%$ of patients with kidney failure were treated for the first time in an acute care hospital. Sixty-three per cent went on to require treatment in an outpatient facility, and only 52\% were alive on therapy at 90 days. The population of ESRD patients continues to grow at a fairly predictable pace and is expected to exceed 11,000 patients by 2011. If current trends continue, $85 \%$ of these individuals will be treated with haemodialysis.

Time series analysis has been used previously by Xue and colleagues (2001) to model the growth in the US ESRD population. Based on data from 1982 to 1997, these authors projected that the incident dialysis population in the United States was likely to grow at an average annual rate of $4.1 \%$ per year, while the prevalent dialysis population was expected to grow at 7.1\% per year between 1997 and 2010. Their models explained greater than $99 \%$ of the variation in outcomes and allowed precise forecasting. The projected growth in incidence and prevalence for the United States was very close to what was observed between 1998 and 2005 in Ontario (4.9\% and $7.2 \%$, respectively). In our study, stepwise auto-regressive models fit incidence and prevalence data well and allowed the most confident projections of the four models employed. In fact, we were able to predict the prevalent dialysis population as far as five years into the future with $95 \%$ confidence limits of $\pm 0.02 \%$.

Following patients from their first dialysis treatment allowed us to study how the definition of chronic dialysis could influence reported incidence and prevalence rates. The point at which an individual becomes a "chronic dialysis patient" is arbitrary. The most commonly used definition is the "90-day rule" developed by the United States Renal Data System (USRDS). Any individual who is alive on renal replacement therapy at 90 days is considered a chronic dialysis patient (USRDS 2006). This definition restricts the population to a stable group of dialysis patients, excluding those who die early in the course of treatment or who have acute, transient renal failure, and has become the standard way of defining ESRD. However, the definition appears to have several drawbacks. Our analysis suggests that the USRDS registry fails to identify almost half of all individuals initiating dialysis therapy. Further, approximately $10 \%$ of patients that will require outpatient dialysis are not captured if patients are not registered until they have been on treatment for 90 days. As a result, prevalence data, and especially incidence rates, may not be comparable to registries that use different definitions of chronic dialysis. Finally, deaths occurring in the first 90 days are not captured, even in patients who truly have ESRD.

Forecasts based on data from national registries are used to make resource allocation decisions. Because individuals treated within the first 90 days of dialysis represent only a small proportion of the prevalent dialysis population (in our case, approximately $3 \%$ ), it could be argued that excluding them from registries is inconsequential. 
However, these patients consume more than the average share of resources (Chertow et al. 2005; Manns et al. 2003). For example, a patient admitted to an intensive care unit with acute renal failure incurs average hospital costs of over $\$ 50,000$ from the start of dialysis until discharge (Manns et al.2003), a figure that approaches the cost of caring for a chronic dialysis patient for an entire year. Further, care of acute patients often diverts resources from individuals receiving chronic dialysis - they require dialysis access (placing a burden on interventional radiology and surgical services) and often, higher nurse-to-patient ratios. In an environment where $62 \%$ of patients receive dialysis for the first time in hospital, these are important considerations. Finally, in dialysis programs that are at or near capacity, underestimating the size of the prevalent population by even $3 \%$ can result in the inability to accommodate new patients (Mendelssohn and Chery 1994). Underestimation has resulted in hospital beds being occupied unnecessarily by stable individuals waiting for a chronic dialysis spot at centres in Ontario.

\section{Limitations}

Our study has limitations. First, the accuracy of OHIP physician billing claims and other administrative databases for identifying dialysis-related variables has not been documented. However, we recently completed a multi-centre validation study that indicates algorithms used in this study to identify demographic variables (age and sex), and the majority of important treatment-related variables are very accurate (started dialysis in hospital, received outpatient dialysis, first treatment modality, first outpatient modality, treatment modality in use at 90 days, and the outcomes of death and renal transplantation). We are also limited by the fact that OHIP claims do not differentiate inpatients who require one-to-one nursing from those who are treated with a normal nurse-to-patient ratio. This is a relevant distinction to administrators of regional dialysis programs that must plan for staffing needs. However, it is likely reliable because nephrologists must submit billing claims in order to be reimbursed for dialysis care.

Second, while time series models fit historical data well and allow precise forecasts of incidence and prevalence, the accuracy of such forecasts is predicated on the persistence of historical trends. This is true of any predictive model and is not unique to time series analysis, but should be considered when interpreting the results.

Third, while growth at a population level has been consistent, modelling growth at a regional level may be more variable.

Fourth, the RPDB tends to overestimate population counts and, as a result, rate calculations may be slightly lower than those obtained using census data. Further, it is important to point out that the rates of incident dialysis reported in this study differ from those reported by the Canadian Organ Replacement Register (CORR), for that 
reason and several others. The exact definition of chronic dialysis used in the CORR registry is somewhat subjective and therefore open to the interpretation of the participating centres. In addition, the CORR reports rates based on a denominator derived from census data and includes all children and adults in its calculations. We restricted our analysis to those 18 years of age and older. Finally, our incident rates include all new dialysis patients in the province, even those who do not meet traditional definitions of chronic dialysis, and therefore appear somewhat higher.

\section{Conclusion}

The annual growth rate in the incidence and prevalence of dialysis is considerable and will present a growing challenge to the healthcare system. Many ESRD registries exclude patients receiving acute dialysis. Although they represent only about $3 \%$ of the prevalent dialysis population, these patients consume many more resources than stable chronic dialysis patients and should be included when planning for future ESRD resource requirements.

\section{ACKNOWLEDGEMENTS}

Robert Quinn is supported by a CIHI Institute for Health Services and Policy Research Fellowship. This study was supported by the Institute for Clinical Evaluative Sciences (ICES), which is funded by an annual grant from the Ontario Ministry of Health and Long-Term Care (MOHLTC). The opinions, results and conclusions reported in this paper are those of the authors and are independent from the funding sources. No endorsement by ICES or the Ontario MOHLTC is intended or should be inferred.

Correspondence may be directed to: Robert R. Quinn, MD, Clinical Associate, Division of Nephrology, Sunnybrook Health Sciences Centre, 2075 Bayview Avenue, Room A239, Toronto, ON M4N 3M5; tel.: 416-480-4755; e-mail: robert. quinn@sunnybrook.ca.

\section{APPENDIX A: BILLING CODES}

Any acute or chronic billing code for traditional, in-centre HD, self-care HD, satellite HD, home HD and nocturnal HD (OHIP billing codes R849, R850, G323, G325, G326, G860, G862, G863, G865 and G866) was classified as "HD." Acute and chronic billing codes for PD included G330, G331, G332, G861 and G864. Finally, all forms of continuous renal replacement therapy were classified as "CRRT" ( G082, G083, G085, G090, G091, G092, G093, G094, G095, G096, G294 and G295, ). 
Renal transplants were identified by Canadian Classification of Procedures (CCP) code 6759 and Canadian Classification of Interventions (CCI) codes 1PC85LAXXJ and 1PC85LAXXK in the CIHI-DAD.

\section{REFERENCES}

Canadian Institute for Health Information (CIHI). 2006. Treatment of End-Stage Organ Failure in Canada, 1995 to 2004. 2006 Annual Report. Ottawa: Author.

Chertow, G.M., E. Burdick, M. Honour, J.V. Bonventre and D.W. Bates. 2005. "Acute Kidney Injury, Mortality, Length of Stay and Costs in Hospitalized Patients." Journal of the American Society of Nephrology 16(11): 3365-70.

De Vecchi, A.F., M. Dratwa and M.E. Wiedemann. 1999."Healthcare Systems and End-Stage Renal Disease (ESRD) Therapies - An International Review: Costs and Reimbursement/ Funding of ESRD Therapies." Nephrology, Dialysis, Transplantation: Official Publication of the European Dialysis and Transplant Association - European Renal Association 14(Suppl. 6): 31-41. Evans, R.W., D.L. Manninen, L.P. Garrison Jr., L.G. Hart, C.R. Blagg, R.A. Gutman, A.R. Hull and E.G. Lowrie. 1985."The Quality of Life of Patients with End-Stage Renal Disease." New England Journal of Medicine 312(9): 553-59.

Farrow, S.C., D.J. Fisher and D.B. Johnson. 1972."Dialysis and Transplantation: The National Picture Over the Next Five Years." British Medical Journal 3(5828): 686-90.

Institute for Clinical Evaluative Sciences (ICES). 2003. Diabetes in Ontario: An ICES Practice Atlas. Toronto: Author.

Laupacis, A., P. Keown, N. Pus, H. Krueger, B. Ferguson, C. Wong and N. Muirhead 1996. “A Study of the Quality of Life and Cost-Utility of Renal Transplantation." Kidney International 50(1): 235-42.

Manns, B., C.J. Doig, H. Lee, S. Dean, M. Tonelli, D. Johnson and C. Donaldson 2003. “Cost of Acute Renal Failure Requiring Dialysis in the Intensive Care Unit: Clinical and Resource Implications of Renal Recovery." Critical Care Medicine 31(2): 449-55.

Mendelssohn, D.C. and A. Chery. 1994. "Dialysis Utilization in the Toronto Region from 1981 to 1992. Toronto Region Dialysis Committee." Canadian Medical Association Journal 150(7): 1099-1105.

Murakami, Y. and Y. Ohashi. 2001."Projected Number of Diabetic Renal Disease Patients among Insulin-Dependent Diabetes Mellitus Children in Japan Using a Markov Model with Probabilistic Sensitivity Analysis." International Journal of Epidemiology 30(5): 1078-83.

Schaubel, D.E., P.G. Blake and S.S. Fenton. 2001."Effect of Renal Center Characteristics on Mortality and Technique Failure on Peritoneal Dialysis." Kidney International 60(4): 1517-24.

US Renal Data System (USRDS). 2006. Annual Data Report: Atlas of End-Stage Renal Disease in the United States. Bethesda, MD: National Institutes of Health, National Institute of Diabetes and Digestive and Kidney Diseases.

Xue, J.L., J.Z. Ma, T.Z. Louis and A. Collins. 2001.“Forecast of the Number of Patients with End-Stage Renal Disease in the United States to the Year 2010." Journal of the American Society of Nephrology 12(12): 2753-58.

You, J., W. Hoy, Y. Zhao, C. Beaver and K. Eagar. 2002."End-Stage Renal Disease in the Northern Territory: Current and Future Treatment Costs." Medical Journal of Australia 176(10): 461-65. 